\title{
Optimized Protocol for PFGE Analysis of Anginosus (milleri) Streptococci.
}

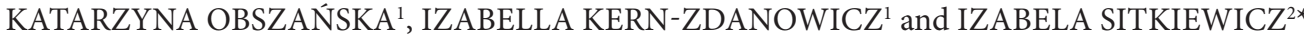 \\ ${ }^{1}$ Department of Microbial Biochemistry, Institute of Biochemistry and Biophysics \\ Polish Academy of Sciences, Warsaw, Poland \\ ${ }^{2}$ Department of Molecular Microbiology, National Medicines Institute, Warsaw, Poland
}

Submitted 9 December 2014, revised 12 December 2014, accepted 24 December 2014

Abstract

Streptococcus anginosus (milleri) is a diverse group of gram positive bacteria. Molecular methods to establish relationship between strains are poorly developed. Therefore, main tool to study genetic variability is restriction fragment length polymorphism combined with pulsed field gel electrophoresis (RFLP-PFGE). In this communication, we present optimized protocol for S. anginosus PFGE analysis.

Key words: Streptococcus anginosus, PFGE

Streptococci are a group of quite diverse gram positive organisms that includes over 40 species (Kohler, 2007). There are multiple methods of classification within this group, often confusing for the non-specialist. The most common classification is simply based on the hemolysis type: full $\beta$ - or partial $\alpha$-hemolysis. However, the classification does not even reflect relationships between species, as the same streptococcal species can sometimes exhibit either $\alpha-, \beta$ - or no hemolysis. The other common classification that uses capital letter designation (groups A, B, C etc.) is based on the presence of specific carbohydrates or lipoteichoic acids on the cell surface, and divides streptococcal species into so called "Lancefield groups" (Lancefield, 1933). This classification, however, can be also confusing as the same Lancefield antigen can be present on the surface of non-related species. In addition species names and evolutionary position also changed over the years (Facklam, 2002). The most recent division into phylogenic groups: pyogenic, anginosus (formerly milleri), mitis/oralis, bovis, salivarius and mutans, reflects evolutionary relationships between species (Kohler, 2007).

The majority of the streptococci that belong to pyogenic group (Streptococcus pyogenes, Streptococcus agalactiae, Streptococcus dysgalactiae subsp. equisimilis) have been studied for years and are well or very well characterized. The same is true for other human pathogens such as Streptococcus pneumoniae. Methods for molecular characterization of these organisms and detecting evolutionary relationships between strains have been described and are widely introduced (Borek et al., 2012a, 2012b; Borek et al., 2011; Obszanska et al., 2012; Obszanska et al., 2011).

Unfortunately, an increasing number of uncharacterized streptococcal species are being regarded as human pathogens. Often, epidemiology and relationships between strains are not studied due to the lack of precise methods.

Rapid, advanced molecular methods to determine relationships between strains, based on known DNA sequences, are usually not developed for less studied groups such as anginosus. Because the anginosus group is poorly characterized at the genetic level, restriction macro-analysis combined with pulse field gel electrophoresis (PFGE) is currently used as the method of choice. Despite the fact that the method is time and labor consuming, it allows to compare strains of unknown characteristics and is regarded as the golden standard in epidemiological research.

PFGE of SmaI (recognized restriction site CCCGGG) digested chromosomal DNA has been for many years the method used to investigate differences between bacterial species/strains with low $\mathrm{G}+\mathrm{C}$ nucleotide content such as streptococci and staphylococci. However, upon inspection of a newly sequenced in our laboratory Streptococcus anginosus strain, we noticed that it contains only few SmaI sites, and generates only 4 distinguishable bands (data not shown). Also, published PFGE analyses show that digest with SmaI yields sometimes as few as 5-6 bands visible on a gel (Bartie et al.,

\footnotetext{
* Corresponding author: I. Sitkiewicz, Department of Molecular Microbiology, National Medicines Institute, Warszawa, Poland; e-mail: isitkiewicz@cls.edu.pl
} 
2000; Chang and Lo, 2013). Therefore we decided to develop a system of macro-restriction PFGE analysis of bacterial strains that belong to the anginosus (milleri) group yielding more bands visible on gel, allowing better resolution and better discriminating between closely related strains. We propose the use of Bsp120I (recognized restriction site GGGCCC), as an less expensive alternative to SmaI, and EagI (recognized restriction site CGGCCG) for more precise differentiation between strains of the anginosus group. We also optimized a step by step procedure of plug preparation and digestion, as well as electrophoresis parameters.

To prepare agarose plugs, strains belonging to the anginosus group should be cultured overnight on Columbia blood agar at $37^{\circ} \mathrm{C}$ in $5 \% \mathrm{CO}_{2}$, then collected using a sterile swab and re-suspended in an ampule of sterile saline. Using densitometer sample density should be adjusted to turbidity 4 on the McFarland scale. One ml of such suspension is then spun in a centrifuge, saline is removed and the pellet re-suspended in $150 \mu \mathrm{l}$ PIV buffer $(1 \mathrm{M} \mathrm{NaCl}, 10 \mathrm{mM}$ Tris- $\mathrm{HCl}$, $\mathrm{pH} 8.0$ ). An equal volume of $2 \%$ SeaPlaque (Lonza) agarose in $\mathrm{ddH}_{2} \mathrm{O}$ pre-warmed to $50^{\circ} \mathrm{C}$ is then added to the bacteria and gently but thoroughly mixed. Next, $20 \mu$ droplets of bacteria in agarose mix are dispensed on a glass plate covered with Parafilm ${ }^{\oplus} \mathrm{M}$ and covered with a microscope slide to form plugs (Chung et al., 2000) and the whole assembly is then placed for $10 \mathrm{~min}$ at $4^{\circ} \mathrm{C}$. When the agarose with embedded bacteria is cooled, agarose droplets are gently moved with the help of a sterile loop into $1.5 \mathrm{ml}$ tubes filled with TE buffer (10 mM Tris-HCl, pH 8.0, 1 mM EDTA, pH 8.0). Plugs can be stored in TE buffer at $4^{\circ} \mathrm{C}$. The period of storing plugs containing whole bacteria, before DNA release, can vary in multiple species and is often a crucial factor to achieve high quality restriction patterns after electrophoresis. However, in the case of anginosus streptococci, we did not notice DNA degradation after keeping undigested plugs in TE for over a year.

To release DNA from bacteria embedded in agarose plugs, one to several plugs can be transferred to $1 \mathrm{ml}$ of EC-lysis buffer in $15 \mathrm{ml}$ conical tubes. EC-lysis buffer must be prepared immediately before use (EC buffer - $6 \mathrm{mM}$ Tris-HCl, pH 8.0, $1 \mathrm{M} \mathrm{NaCl}, 0.1 \mathrm{M}$ EDTA, $\mathrm{pH} 8.0,0.2 \%$ sodium deoxycholate, $0.5 \%$ sodium lauroyl sarcosinate, $0.5 \%$ Brij $^{\varpi} 58$; EC-lysis buffer - EC buffer with addition of $1 \mathrm{mg} / \mathrm{ml}$ lysozyme, $0.1 \mathrm{mg} / \mathrm{ml}$ RNase, $100 \mathrm{U} / \mathrm{ml}$ mutanolysin). Plugs in EC-lysis buffer should be incubated for 4 hours at $37^{\circ} \mathrm{C}$. After incubation, the buffer is removed and replaced with $1 \mathrm{ml}$ of ES buffer (ES buffer - 0.5 M EDTA, pH 9.0, 1\% sodium lauroyl sarcosinate) and $1 \mathrm{mg} / \mathrm{ml}$ of proteinase $\mathrm{K}$ and the tubes are left overnight at $50^{\circ} \mathrm{C}$.

After overnight incubation, ES buffer with proteinase is removed and two $7 \mathrm{ml}$ washes with TE are performed. For each wash plugs must be incubated for 30 min with gentle mixing on an orbital shaker at room temperature. After the second wash, TE is replaced with $1 \mathrm{ml}$ TE buffer with $20 \mu \mathrm{l} 0.1 \mathrm{M}$ PMSF and incubate $30 \mathrm{~min}$ at room temperature without shaking. After incubation with TE + PMSF three additional washes with $10 \mathrm{ml}$ of TE are performed.

Washed plugs with released DNA can be digested immediately with restriction enzymes, however, plugs with released DNA can be stored in TE buffer. We recommend that plugs are used for restriction digest and electrophoresis as soon as it is possible, but we did not observe markedly visible loss of quality even after 4 months of storage in TE buffer.

To perform the digest, one plug should be placed in $1.5 \mathrm{ml}$ tube, filled with $100 \mu \mathrm{l}$ of appropriate $1 \times$ digestion buffer and incubated for $30 \mathrm{~min}$ at $37^{\circ} \mathrm{C}$. After

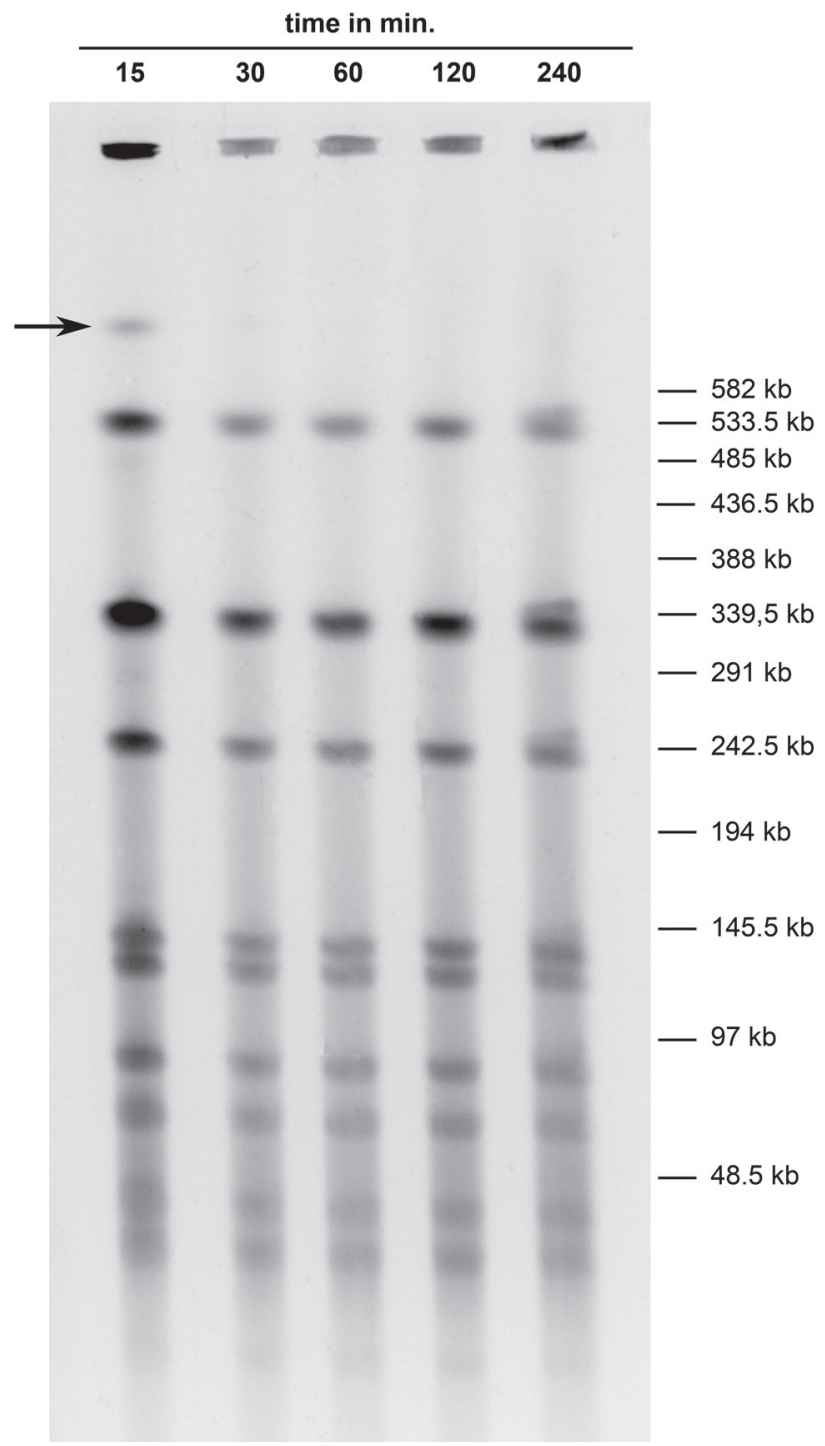

Fig. 1. Majority of chromosomal DNA released from plugs is digested after 15 minutes of incubation with $0.5 \mu$ l of FastDigest ${ }^{\circledast}$ Bsp120I restriction enzyme. Incompletely cut DNA is marked by a black arrow. Marker - Lambda Ladder PFG Marker (New England Biolabs) 
that time the buffer should be replaced with $50 \mu$ of diluted Bsp120I or EagI $(49.5 \mu$ l of the $1 \times$ restriction buffer $+0.5 \mu$ of the enzyme). Digestion time should be optimized, however, we observed that FastDigest ${ }^{\circledast}$ restriction enzymes (available from Thermo Scientific/ Fermentas) used in this protocol can noticeably shorten the whole procedure. As can be seen in Figure 1 for Bsp120I, chromosomal DNA can be digested almost completely using a single $0.5 \mu \mathrm{l}$ aliquot of the enzyme within 15 minutes. Only a small amount of uncut DNA (marked by an arrow) is visible on a gel. An hour long incubation with $B s p 120$ I always presented completely digested DNA. We usually digested our samples with $0.5 \mu \mathrm{l}$ of EagI for four hours for complete digest, because of more restriction sites usually present in the genome.

Digestion with both enzymes yields a substantial (10-28) number of bands that can be used to differentiate between strains. Bsp120I is a cheap alternative to other restriction enzymes, yet a considerable set of anginosus group strains is not digested by Bsp120I. We tested whether this is caused by poorly digested cell wall and unreleased DNA. As a control we used the same lot of plugs for both Bsp120I and EagI digestion and we observed that DNA in plugs not digested by Bsp120I was digested by EagI (Fig. 2).

Because Bsp120I is an enzyme blocked by Dcm methylation, we further tested whether this type of methylation affects digestion of anginosus group strains DNA released from plugs. To assess the influence of
Dcm methylation on Bsp120I activity, we digested chromosomal DNA isolated from strains whose DNAs were unable to be digested with Bsp120I, with EcoRII and $M v a I$. EcoRII and MvaI recognize the same DNA sequence but are sensitive and non-sensitive to $\mathrm{Dcm}$ methylation, respectively. We observed that both enzymes digested DNA (data not shown), so the inability of Bsp120I to digest DNA released from anginosus group is not related to Dcm methylation. Therefore, we hypothesize that we may observe a similar phenomenon as in the case of SmaI digestion of Staphylococcus aureus DNA (Bens et al., 2006). SmaI, but not its neoschizomers such as $\mathrm{XmaI}$ or $\mathrm{Cfr}$ II, is blocked by the presence of 5-methylcytosine at specific sites in its recognition sequence CCCGGG. It was noticed that a particular line of $S$. aureus MRSA strains cannot be analyzed by PFGE using standard SmaI restrictase due to the activity of a specific uncharacterized restriction/ methylation system present in this MRSA clone. We suspect that GGGCCC restriction site recognized by Bsp120I in anginosus group can be also influenced by an uncharacterized so far methylation system.

The electrophoresis was run in $1 \%$ SeaKem ${ }^{\oplus}$ Gold agarose (Lonza) gel in standard electrophoresis $0.5 \times$ TBE buffer. Prior to the run the gel was placed in the electrophoresis chamber of a CHEF Mapper ${ }^{\otimes}$ XA Pulsed Field Electrophoresis System (BioRad), covered with $0.5 \times \mathrm{TBE}$ buffer and chilled to $11^{\circ} \mathrm{C}$. Digested plugs were washed with TBE buffer, placed in wells, one

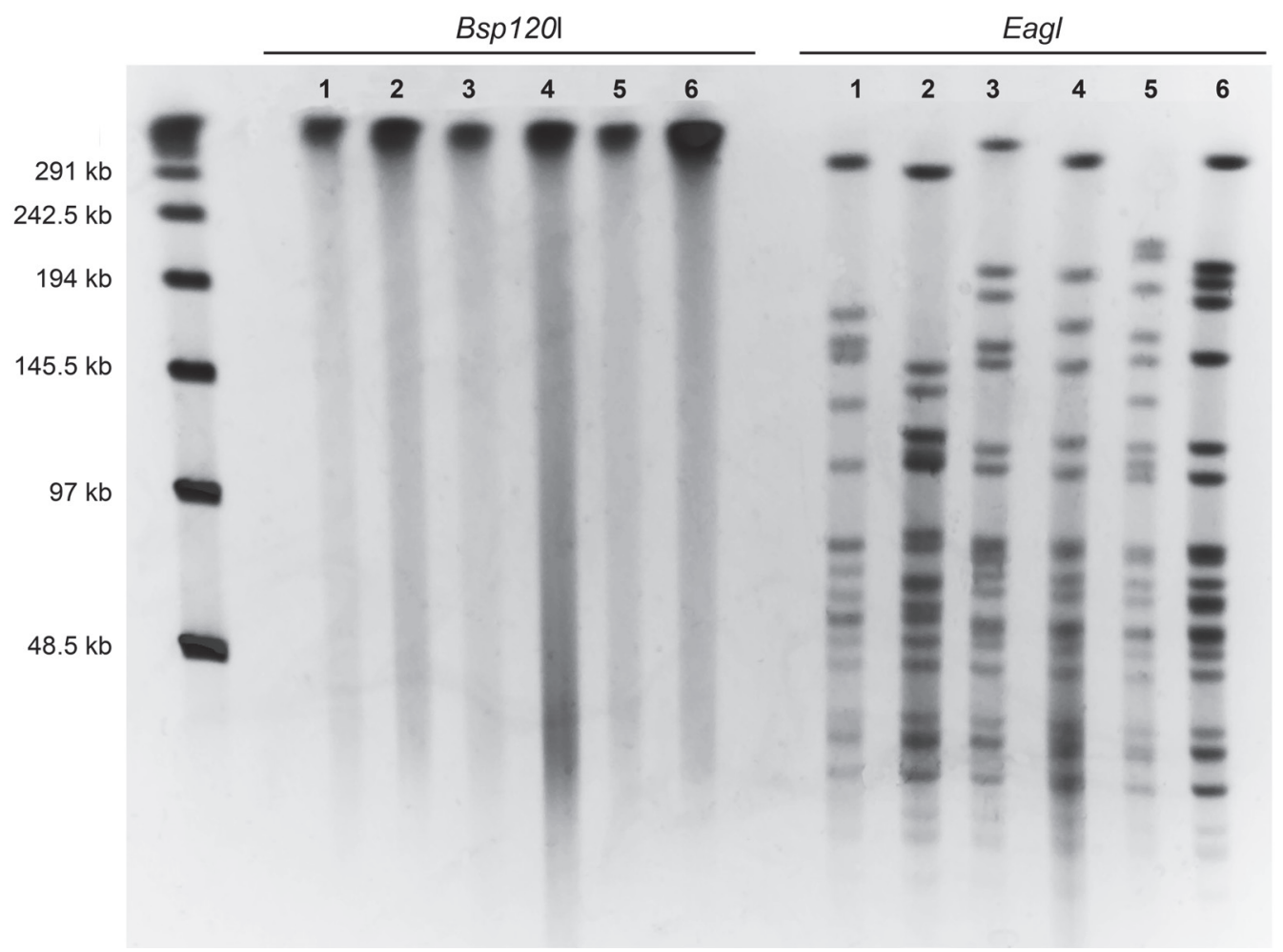

Fig. 2. Chromosomal DNA released from the same lots of plugs (marked 1 through 6) cannot be digested with Bsp120I, but is cut by EagI. Marker - Lambda Ladder PFG Marker (New England Biolabs). 
plug per well, and covered with $1 \%$ SeaKem agarose. Fragments were separated for 23 hours at $6 \mathrm{~V} / \mathrm{cm}$ using a $120^{\circ}$ included angle. For Bsp120I initial switch time was $2 \mathrm{~s}$ and final switch time $50 \mathrm{~s}$, for EagI the initial switch time was $1 \mathrm{~s}$ and the final switch time $20 \mathrm{~s}$. After electrophoresis, the gel was stained in $0.5 \times \mathrm{TBE}$ buffer with $0.5 \mu \mathrm{g} / \mathrm{ml}$ of ethidium bromide for $\sim 45$ minutes with gentle shaking and photographed under UV light. Gels can be kept overnight in staining solution at $4^{\circ} \mathrm{C}$. Prolonged staining increases the level of band detection sensitivity and produces images with less background. Also, to decrease the background, stained gels can be briefly de-stained $(2 \times 5$ minutes $)$ with water.

As a final step PFGE patterns on single or multiple gels can were compared using Tenover criteria (Tenover et al., 1995) or various algorithms dedicated to band pattern clustering and dendrogram construction such as BioNumerics software (Applied Maths).

\section{Acknowledgments}

The work was financed by the grant from National Center for Science (NCN) number NN401535940.

We would like to thank dr Janusz Fiett and dr Radosław Izdebski for valuable discussions and help with electrophoresis optimization.

\section{Literature}

Bartie K.L., M.J. Wilson, D.W. Williams and M.A. Lewis. 2000. Macrorestriction fingerprinting of "Streptococcus milleri" group bacteria by pulsed-field gel electrophoresis. J. Clin. Microbiol. 38: 2141-2149.

Bens C.C., A. Voss and C.H. Klaassen. 2006. Presence of a novel DNA methylation enzyme in methicillin-resistant Staphylococcus aureus isolates associated with pig farming leads to uninterpretable results in standard pulsed-field gel electrophoresis analysis. J. Clin. Microbiol. 44: 1875-1876.

Borek A.L., J. Wilemska, R. Izdebski, W. Hryniewicz and I. Sitkiewicz. 2011. A new rapid and cost-effective method for detection of phages, ICEs and virulence factors encoded by Streptococcus pyogenes. Pol. J. Microbiol. 60: 187-201.

Borek A.L., K. Obszanska, W. Hryniewicz and I. Sitkiewicz. 2012a. Detection of Streptococcus pyogenes virulence factors by multiplex PCR. Virulence 3: 529-533.

Borek A.L., K. Obszanska, W. Hryniewicz and I. Sitkiewicz. 2012b. Typing of Streptococcus pyogenes strains using the phage profiling method. Virulence 3: 534-538.

Chang Y.C. and H.H. Lo. 2013. Identification, clinical aspects, susceptibility pattern, and molecular epidemiology of beta-haemolytic group G Streptococcus anginosus group isolates from central Taiwan. Diagn. Microbiol. Infect. Dis. 76: 262-265.

Chung M., H. de Lencastre, P. Matthews, A. Tomasz, I. Adamsson, M. Aires de Sousa, T. Camou, C. Cocuzza, A. Corso, I. Couto and others. 2000. Molecular typing of methicillin-resistant Staphylococcus aureus by pulsed-field gel electrophoresis: comparison of results obtained in a multilaboratory effort using identical protocols and MRSA strains. Microb. Drug. Resist. 6: 189-198.

Facklam R. 2002. What happened to the streptococci: overview of taxonomic and nomenclature changes. Clin. Microbiol. Rev. 15: 613-630.

Kohler W. 2007. The present state of species within the genera Streptococcus and Enterococcus. Int. J. Med. Microbiol. 297: 133-150.

Lancefield R.C. 1933. A serological differentiation of human and other groups of hemolytic streptococci. J. Exp. Med. 57: 571-595.

Obszanska K., A.L. Borek, R. Izdebski, W. Hryniewicz and I. Sitkiewicz. 2011. Multilocus variable number tandem repeat analysis (MLVA) of Streptococcus pyogenes. J. Microbiol. Methods. 87: 143-149. Obszanska K., A.L. Borek, W. Hryniewicz and I. Sitkiewicz. 2012. Multiple locus VNTR fingerprinting (MLVF) of Streptococcus pyogenes. Virulence 3: 539-542.

Tenover F.C., R.D. Arbeit, R.V. Goering, P.A. Mickelsen, B.E. Murray, D.H. Persing and B. Swaminathan. 1995. Interpreting chromosomal DNA restriction patterns produced by pulsed-field gel electrophoresis: criteria for bacterial strain typing. J. Clin. Microbiol. 33: 2233-2239. 